\title{
Low CCL17 expression associates with unfavorable postoperative prognosis of patients with clear cell renal cell carcinoma
}

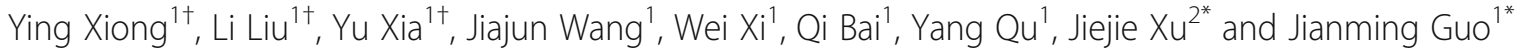

\begin{abstract}
Background: Chemokine (C-C motif) ligand 17 (CCL17) is a chemokine mainly produced by myeloid dendritic cells. It is a ligand for CC chemokine receptor 4 (CCR4) and CC chemokine receptor 8 (CCR8). The aim of this study was to investigate prognostic values of CCL17 expression in patients with clear cell renal cell carcinoma (cCRCC).

Methods: The study included 286 patients with cCRCC. CCL17 expression was analyzed by immunohistochemistry on tissue microarrays. Prognostic values of CCL17 expression and patients' clinical outcomes were evaluated.

Results: Kaplan-Meier method showed that low CCL17 expression was associated with worse patient overall survival $(\mathrm{OS})$ and recurrence-free survival (RFS) (OS, $P=0.002 ; \mathrm{RFS}, P=0.007)$. Low CCL17 expression was an adverse independent risk factor for OS and RFS in multivariate analyses (OS, $P=0.006, P=0.011$ for bootstrap; RFS, $P=0.002, P=0.025$ for bootstrap). We constructed two nomograms incorporating parameters derived from multivariate analyses to predict patients' OS and RFS (OS, c-index 0.799; RFS, c-index 0.787) and they performed better than existed integrated models.

Conclusion: Low CCL17 expression is a potential independent adverse prognostic biomarker for recurrence and survival of patients with ccRCC after nephrectomy. Established nomograms based on this information could help predict ccRCC patients' OS and RFS.
\end{abstract}

Keywords: Chemokine (C-C motif) ligand 17, Clear cell renal cell carcinoma, Prognostic factor, Overall survival, Recurrence-free survival

\section{Background}

Renal cell carcinoma (RCC) is the most common malignant cancer in the adult kidney and accounts for 2 to $3 \%$ of all malignancies in adults [1]. Clear-cell RCC (ccRCC) is the major histological subtype according to the WHO classification, which accounts for $80-90 \%$ of all RCC patients [2]. Around one third of the patients who underwent curative surgeries would develop recurrences or metastases afterwards [3]. Several prognostic factors and integrated staging systems have been developed for RCC patients such as TNM stage, Fuhrman grade and several integrated models like University of California Integrated

\footnotetext{
* Correspondence: jjxufdu@fudan.edu.cn; guo.jianming@zs-hospital.sh.cn ${ }^{\dagger}$ Equal contributors

${ }^{2}$ Department of Biochemistry and Molecular Biology, School of Basic Medical Sciences, Fudan University, Shanghai 200032, China

${ }^{1}$ Department of Urology, Zhongshan Hospital, Fudan University, Shanghai 200032, China
}

Staging System (UISS) and Mayo Clinic stage, size, grade and necrosis (SSIGN) score [4]. Unfortunately, these models are not accurate enough due to the genetic complexity and heterogeneity of the disease [5]. Improved predictive models of survival for ccRCC are needed.

Numerous evidences indicate that chemokines play pleiotropic roles in tumor cell biology [6]. Chemokine (C-C motif) ligand 17 (CCL17), also known as thymus and activation-regulated chemokine (TARC) [7], is a chemokine produced by myeloid dendritic cells, endothelial cells, bronchial epithelial cells and several tumor cells [8]. It is a ligand for CC chemokine receptor 4 CR4) and CC chemokine receptor 8 (CCR8). It is able recruit $\mathrm{T}$ cells particularly Th2 cells and activate other tigen-presenting cells [9]. Researchers recently proved that CCL17 was involved in recruiting cytotoxic T cells by binding to CCR4 [10] and activating CD8+ T cells 
through dendritic cells [11]. These findings indicate that CCL17 is able to enhance antitumor immunity. We wondered whether chemokine CCL17 could act as a promising biomarker candidate for RCC. The role of CCL17 in the development of ccRCC remains unknown so we analyzed the impact of CCL17 expression on patients' overall survival (OS) and recurrence-free survival (RFS) in a large cohort of ccRCC patients.

\section{Methods}

\section{Patients and specimens}

A total of 286 ccRCC patients who received nephrectomy in Zhongshan Hospital, Fudan University during Jan 2005 and Jun 2007 were enrolled in our study. Clinical Research Ethics Committee of Zhongshan Hospital, Fudan University had approved the study and granted permissions to access the patient records. Written and informed consent was obtained from each individual enrolled in the study. Clinicopathological variables included age, gender, tumor size, TNM stage, Fuhrman grade, Eastern Cooperative Oncology Group performance status (ECOG PS) and necrosis. SSIGN score and UISS score were assessed for each patient. Patients should meet the primary criteria of having pathologically proved ccRCC, having received nephrectomy and having available Formalin Fixed Paraffin Embedded (FFPE) specimen of tumor mass $\left(\geq 1 \mathrm{~cm}^{3}\right)$. Patients who had other former malignant tumors, perioperative mortalities, histories of adjuvant or neo-adjuvant therapies including targeted therapies, mixed type renal cancer or bilateral renal cancer were excluded. Samples with over $80 \%$ necrotic or hemorrhagic area were excluded either.

\section{Data collection}

Patients' OS was defined as the time of nephrectomy to the time of death or last follow up while RFS was calculated from the time of nephrectomy to the time of recurrence. Recurrence was confirmed by imaging, biopsy or physical examination. There were altogether 24 patients excluded from RFS analysis because of missing data of recurrence state or preoperational metastases. Patients were followed up every 3 months during the first 5 years after operation and once a year thereafter. Data was censored until Jan 30, 2015, the last follow up time or the time when patient died. Two pathologists (Yuan J. and Jun $H$.) reviewed the H\&E slides to reconfirm histological subtype, stage, and Fuhrman grade. They confirmed ccRCC histological subtypes according to the 2014 EAU guidelines [2]. Tumor stage was classified according to the 2010 AJCC TNM classification [12]. Fuhrman grade and necrosis were reported according to 2012 ISUP consensus [13]. The SSIGN, UISS and SSIGN localized (Leibovich) score were applied to stratify patients into different risk groups [14-16].

\section{Immunohistochemistry and evaluation}

We constructed tissue microarrays (two cores for one tumor block) with formalin-fixed, paraffin embedded surgical specimens. Immunohistochemical staining was performed on tissue microarrays with protocols described previously [17]. Antibodies against CCL17 (Anti-TARC antibody, ab182793, Abcam, diluted 1/100) and visualization reagent (DakoEnVision Detection System) were used. The specificity of the antibody was confirmed by western blot using RCC cell lines. We used Olympus CDD camera, Nikon eclipse Ti-s microscope $(\times 200$ magnification and $\times 400$ magnification $)$ and NIS-Elements F3.2 software to record the staining results. We took three independent shots and chose the strongest for each tumor core. The intensity of immunohistochemical staining of CCL17 was scored by two urologists unaware of the patients' clinical features and outcomes using Image-Pro Plus version6.0 software (Media Cybernetics Inc., Bethesda, MD, USA). The pooled IOD mean of the six spots in two tumor cores was regarded as the final staining intensity for each block. We defined IOD score of 8461 as the cutoff value for high and low expression with $\mathrm{X}$-tile software according to the 'minimum $P$-value method' on the basis of its relation with OS [18].

\section{Statistical analyses}

Statistical analyses were performed using SPSS Statistics 21.0 (SPSS Inc., Chicago, IL), R software version 3.0.2 with the "rms", "smoothHR" and "phenoTest" [19] package ( $\mathrm{R}$ Foundation for Statistical Computing, Vienna, Austria) and Stata (version 12.1; StataCorp LP, TX, USA). Mann-Whitney $U$ test, Pearson's chi-square test, Fisher's exact test or Cochran-Mantel-Haenszel $\chi^{2}$ test was used to compare clinicopathological parameters of the patients. Kaplan-Meier analysis was applied to plot the survival curve. Log-rank test was used to compare patient survival between subgroups. Log-rank $P$ values were corrected using the formula proposed by Altman and colleagues [20] since $P$ values obtained through "minimum $p$ value method" might be overestimated. Numbers at risk were calculated at the beginning of each time period. The Cox proportional hazards regression model was used to perform univariate and multivariate analyses. Besides, 1000 bootstrap resamples were performed for reducing overfitting bias. Two nomograms were constructed to predict the OS and RFS. We calculated concordance index to compare the prognostic or predictive accuracy of different models. Hanley-McNeil test was applied to compare the difference between c-indexes. All statistical tests were 2 -sided and $P<0.05$ was considered statistically significant. 


\section{Results}

\section{Expression of CCL17 and its correlation with} clinicopathological characteristics

We first evaluated CCL17 expression by immunohistochemistry staining analysis in 286 ccRCC patients. CCL17 expression was predominantly found on the cytoplasm of tumor cells and the intensity of the staining was variable (Fig. 1a and b). We illustrated the smooth estimated HR of CCL17 expression (+1 IOD score) on patients' OS (Fig. 1c). According to the cutoff value (8461) derived from IOD scores and "minimum $p$ value method", 143 of 286 patients were assigned to the low CCL17 expression group and others were assigned to the high CCL17 expression group. The smooth HR curve displayed a significant and stable prognostic difference between the high and low CCL17 expression patient groups with the cutoff value as a reference (Fig. 1d). The curve of smooth estimates did not fluctuate much and log hazard ratio kept decreasing with the increase of IOD scores of CCL17 expression, which indicated that a two-level classification was appropriate (Fig. 1c). CCL17 expression was significantly associated with ECOG PS $(P=0.003)$ while its correlation with other clinicopathological characteristics did not meet statistical significance. The associations between CCL17 expression and clinicopathological features were summarized in Table 1. The median follow-up time was 90.87 months (range 2.63-120.47).

\section{High CCL17 expression is associated with better prognosis}

Kaplan-Meier survival analysis was performed to compare OS and RFS according to CCL17 expression. $P$ values were corrected [20]. Patients with high CCL17 expression had a significantly better OS $(P=0.002)$ and RFS $(P=0.007)$ than patients with low CCL17 expression (Fig. 2a and d). We then performed univariate and multivariate analyses to further assess whether CCL17 expression was an independent prognostic factor of OS and RFS. Univariate analysis showed that IOD score as a continuous viable was significantly associated with OS and RFS. CCL17 expression as a dichotomous variable was also a risk factor for both OS and RFS in univariate analysis (Additional file 1: Table S1). Furthermore, in multivariate analysis, high CCL17 expression was also a favorable independent risk factor for both OS and RFS (OS, HR, 0.504, 95\% CI, 0.309-0.824, $P=0.006, P=0.011$ for bootstrap; RFS, HR, 0.448, 95\% CI, 0.267-0.751, $P=$ $0.002, P=0.025$ for bootstrap). Pathological $\mathrm{T}$ stage,

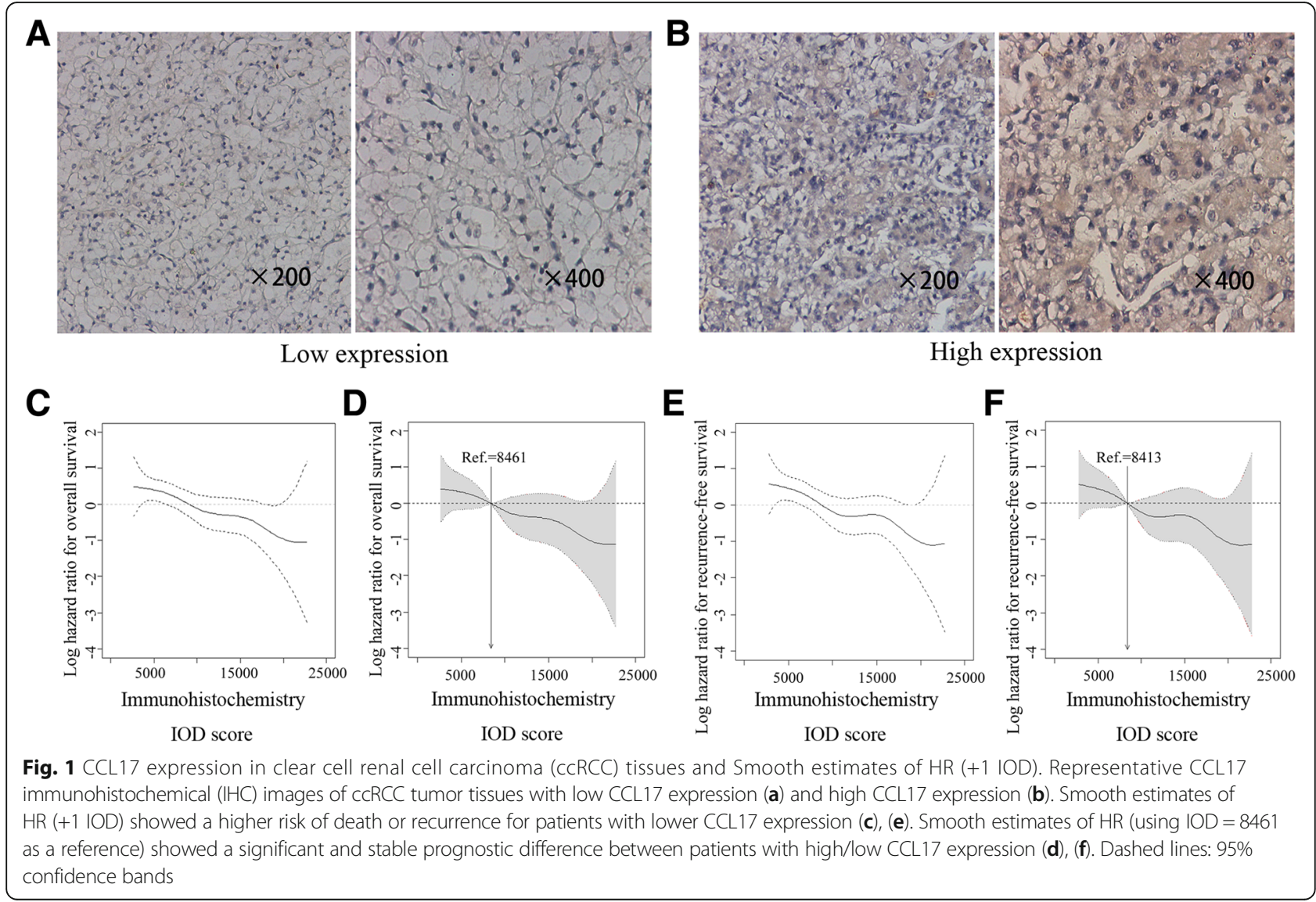


Table 1 Clinical characteristics of patients according to CCL17 expression

\begin{tabular}{|c|c|c|c|c|c|}
\hline \multirow[t]{2}{*}{ Characteristics } & \multicolumn{2}{|c|}{ Patients } & \multicolumn{3}{|c|}{ CCL17 expression } \\
\hline & $\mathrm{n}$ & $\%$ & Low & High & $P$-value \\
\hline All patients & 286 & 100 & 143 & 143 & \\
\hline Age, years & & & & & $0.811^{\mathrm{a}}$ \\
\hline mean $\pm S D$ & 55.37 & 3.24 & $55.69 \pm 13.46$ & $55.05 \pm 13.06$ & \\
\hline Gender & & & & & $0.368^{b}$ \\
\hline Female & 87 & 30.4 & 47 & 40 & \\
\hline Male & 199 & 69.6 & 96 & 103 & \\
\hline Tumor size, cm & & & & & $0.173^{\mathrm{a}}$ \\
\hline mean $\pm S D$ & 4.81 & & $5.04 \pm 2.76$ & $4.58 \pm 2.56$ & \\
\hline Pathological T stage & & & & & $0.301^{c}$ \\
\hline pT1 & 181 & 63.3 & 85 & 96 & \\
\hline pT2 & 26 & 9.1 & 16 & 10 & \\
\hline pT3 & 75 & 26.2 & 40 & 35 & \\
\hline pT4 & 4 & 1.4 & 2 & 2 & \\
\hline Pathological N stage & & & & & $1.000^{\mathrm{b}}$ \\
\hline $\mathrm{pNx}$ & 240 & 83.9 & 120 & 120 & \\
\hline pNO & 44 & 15.4 & 22 & 22 & \\
\hline pN1 & 2 & 0.7 & 1 & 1 & \\
\hline Distant metastasis & & & & & $0.063^{b}$ \\
\hline No & 271 & 94.8 & 132 & 139 & \\
\hline Yes & 15 & 5.2 & 11 & 4 & \\
\hline TNM stage & & & & & $0.122^{c}$ \\
\hline । & 175 & 61.2 & 79 & 96 & \\
\hline$\|$ & 23 & 8.0 & 13 & 10 & \\
\hline III & 69 & 24.1 & 35 & 34 & \\
\hline IV & 19 & 6.6 & 14 & 5 & \\
\hline Fuhrman grade & & & & & $0.456^{c}$ \\
\hline 1 & 32 & 11.2 & 17 & 15 & \\
\hline 2 & 209 & 73.1 & 99 & 110 & \\
\hline 3 & 41 & 14.3 & 25 & 16 & \\
\hline 4 & 4 & 1.4 & 2 & 2 & \\
\hline Necrosis & & & & & $0.866^{\mathrm{b}}$ \\
\hline Absent & 245 & 85.7 & 123 & 122 & \\
\hline Present & 41 & 14.3 & 20 & 21 & \\
\hline ECOG PS & & & & & $0.003^{b}$ \\
\hline 0 & 208 & 72.7 & 93 & 115 & \\
\hline$\geq 1$ & 78 & 27.3 & 50 & 28 & \\
\hline UISS category & & & & & $0.277^{c}$ \\
\hline Low risk & 119 & 41.6 & 54 & 65 & \\
\hline Mediate risk & 127 & 44.4 & 63 & 64 & \\
\hline High risk & 40 & 14.0 & 24 & 16 & \\
\hline SSIGN category & & & & & $0.143^{c}$ \\
\hline $0-3$ & 218 & 76.2 & 105 & 113 & \\
\hline $5-7$ & 62 & 21.8 & 33 & 29 & \\
\hline $8+$ & 6 & 2.1 & 5 & 1 & \\
\hline
\end{tabular}

ECOG PS Eastern Cooperative Oncology Group performance status $P$-value $<0.05$ was regarded as statistically significant ${ }^{a}$ Mann-Whitney $U$ test for continuous variables, ${ }^{b} X^{2}$ test or Fisher's exact test ${ }^{c}$ Cochran-Mantel-Haenszel $x^{2}$ test distant metastasis, necrosis, Fuhrman grade and ECOG PS were significantly associated with OS and RFS (Table 2).

\section{Extension of Prognostic Model with CCL17 expression for CCRCC}

As is shown in Fig. 2, the SSIGN/SSIGN (localized) score was applied to classify patients into two risk levels: $0-3$ (low), $\geq 4$ (mediate and high) for OS analysis and $0-2$ (low), $\geq 3$ (mediate and high) for RFS analysis. High CCL17 expression displayed as a good prognostic factor in intermediated- and high-risk groups in both OS and RFS analyses (OS, $P=0.004$; RFS, $P=0.006$ ). Then, we sought to investigate whether the incorporation of the CCL17 expression into TNM stage, UISS, and SSIGN would improve their predictive accuracy by calculating the C-index (Table 3). CCL17 expression information could add additional power into several existed RCC prognostic models (OS, $P=0.003$, RFS, $P=0.002$ for TNM; OS, $P=0.006$, RFS, $P=0.001$ for SSIGN; OS, $P<0.001$, RFS, $P=0.001$ for UISS) (Table 3 ).

\section{Prognostic Nomograms of ccRCC}

We constructed two nomograms to predict OS and RFS at 5 and 8 years after nephrectomy (Fig. 3a, d). The predictors were based on the validated multivariate analyses (Table 2), including pathological $\mathrm{T}$ stage, distant metastasis, Fuhrman grade, necrosis status, ECOG PS and CCL17 expression. The C-index showed a good predictive accuracy for nomograms in both OS and RFS (OS, Cindex 0.799; RFS, C-index 0.787). The nomogram to predict OS showed better prognostic capability compared with SSIGN (Nomogram vs SSIGN, $P<0.001$ ) and UISS (Nomogram vs UISS, $P<0.001$ ). In terms of RFS, the second nomogram also performed better than SSIGN (Nomogram vs SSIGN, $P<0.001$ ) and UISS (Nomogram vs UISS $P=0.001$ ). Calibration curves for nomogram predicted 5-year and 8-year OS (Fig. 3b, c) and RFS (Fig. 3e, f) were established and the plots displayed good consistency between the predicted and actual observation of patients' survival.

\section{Discussion}

In this study, we found that CCL17 was predominantly expressed on cytoplasm of tumor cells through immunochemistry, and high CCL17 expression turned out to be positively correlated with a better prognosis. What's more, CCL17 expression was an independent prognostic factor for OS and RFS of ccRCC patients. The combination of CCL17 expression and current prognostic models like TNM stage, UISS and SSIGN was able to enhance prognostic accuracy. In the end, we generated two nomograms by incorporating CCL17 with other clinicopathological parameters derived from multivariate analysis to predict patients' OS and RFS. Comparisons by C-indexes showed 


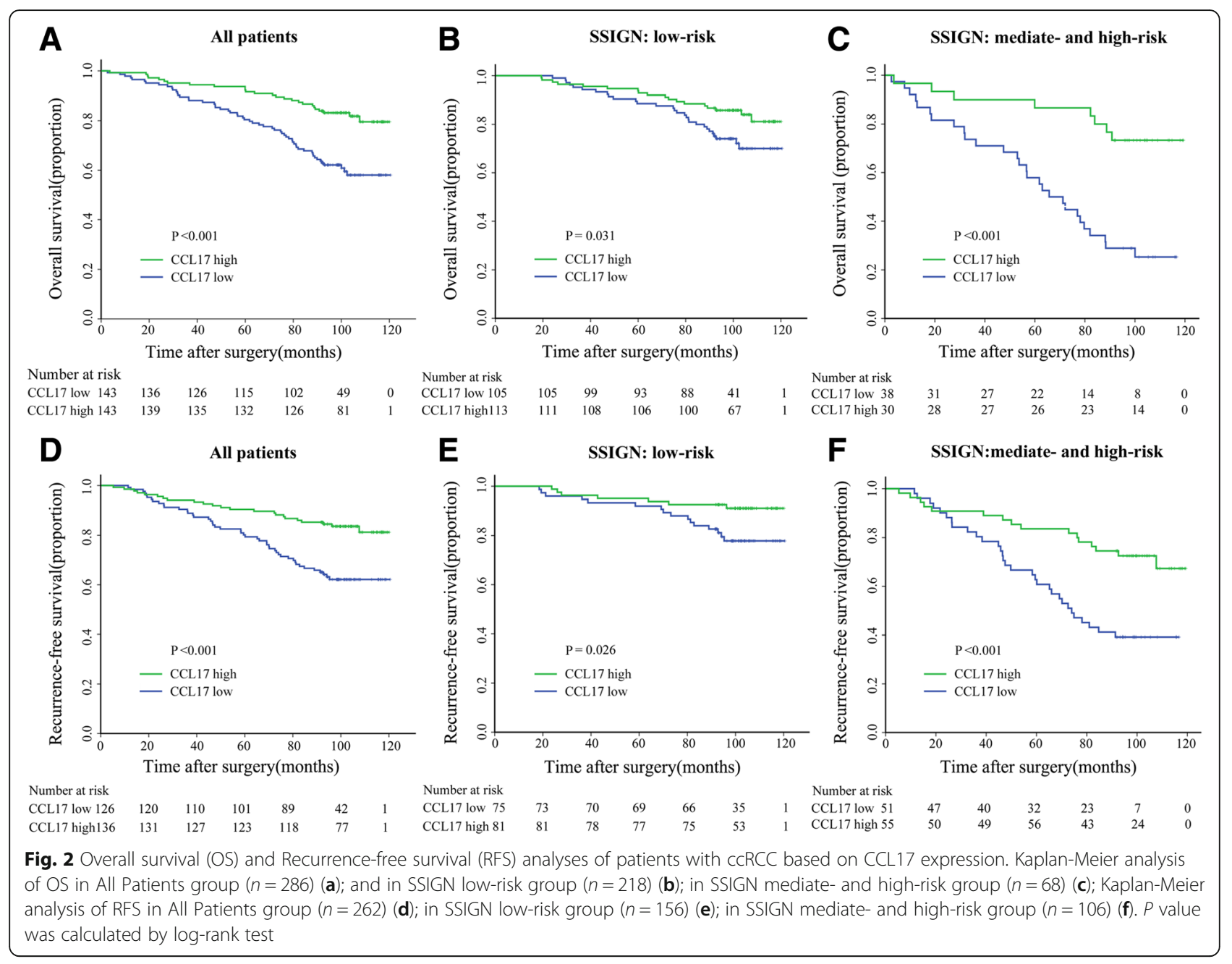

that the two nomograms performed better than current prognostic models.

As a member of the CC-motif chemokine family, CCL17 is actively secreted by immune cells. CCL17 binds to the G-protein coupled CCR4 and CCR8 and serves for the recruitment and migration of these receptor-expressing cells $[21,22]$. CCL17 was considered to attract CCR4+ Treg cells to the tumor. CCL17 created a favorable environment where tumor cells could escape from host immune responses in some type of cancers [23]. Different functions of CCL17 are being discovered these years. CCL17 produced by dendritic cells is able to attract naive cytotoxic $\mathrm{T}$ lymphocytes expressing CCR4 and enhance cytotoxicity [10]. It is also a mediator of CD8 $+\mathrm{T}$ cell activation through dendritic cells [11]. The prognostic significance of CCL17 varies with the type of malignancy. CCL17 high expression in tumor cells predicts poor survival in patients with hepatocellular carcinoma [24]. Elevated serum level of CCL17 predicts better survival in renal cell carcinoma after peptide vaccination [25] and melanoma carcinoma [26]. Researchers treated 68 subjects with IMA901, a therapeutic vaccine for RCC consisting of multiple tumor associated peptides. Among 300 serum biomarkers, researchers identified that low apolipoprotein A-I and CCL17 predicted worse IMA901 treatment response and overall survival [25]. Our study revealed that low CCL17 expression was also associated with poor patients' survival without IMA901 treatment.

CCR4 is expressed by CD4+ T cells, CD8+ cytotoxic T lymphocytes, natural killer cells, macrophages and subsets of DCs [27]. Mogamulizumab (KW-0761) is a humanized antiCCR4 mAb approved for treatment of certain types of adult T-cell leukemia and peripheral Tcell lymphoma. A clinical study of mogamulizumab for the treatment of CCR4-negative advanced or recurrent solid cancer is now conducted [28]. CC17 and CCL22 are both ligands of CCR4 but they probably have opposing effects on Treg homeostasis in that CCL22 favors Treg recruitment whereas CCL17 has been reported to convert the Treg phenotype to an inflammatory mediator $[29,30]$. 
Table 2 Proportional hazard model for overall survival and recurrence-free survival prediction

\begin{tabular}{|c|c|c|c|c|c|c|}
\hline \multirow[t]{2}{*}{ Variables } & \multicolumn{3}{|l|}{ OS $(n=286)$} & \multicolumn{3}{|l|}{ RFS $(n=262)$} \\
\hline & HR $(95 \% \mathrm{Cl})$ & $P$-valuet & $\begin{array}{l}p \text {-valuet } \\
\text { (bootstrap } \ddagger)\end{array}$ & HR $(95 \% \mathrm{Cl})$ & $P$-valuet & $\begin{array}{l}p \text {-valuet } \\
\text { (bootstrap } \neq)\end{array}$ \\
\hline Pathological T stage & & $<0.001$ & & & $<0.001$ & \\
\hline pT1 & Reference & & & Reference & & \\
\hline pT2 & $2.655(1.361-5.180)$ & 0.004 & 0.005 & $2.586(1.197-5.587)$ & 0.016 & 0.011 \\
\hline pT3 & $2.879(1.756-4.720)$ & $<0.001$ & 0.001 & $3.061(1.795-5.221)$ & $<0.001$ & 0.002 \\
\hline pT4 & 3.809 (1.054-14.422) & 0.027 & 0.292 & $8.842(2.712-28.823)$ & $<0.001$ & 0.009 \\
\hline \multicolumn{7}{|l|}{ Distant metastasis } \\
\hline Yes vs No & $2.467(1.283-4.744)$ & 0.007 & 0.073 & & & \\
\hline Fuhrman grade & & 0.005 & & & 0.001 & \\
\hline 1 & Reference & & & Reference & & \\
\hline 2 & $1.957(0.701-5.466)$ & 0.200 & 0.219 & $1.439(0.563-3.674)$ & 0.447 & 0.490 \\
\hline 3 & 4.067 (1.353-12.223) & 0.012 & 0.012 & $4.024(1.433-11.301)$ & 0.008 & 0.019 \\
\hline 4 & 6.786 (1.494-30.828) & 0.013 & 0.002 & $5.377(1.258-22.981)$ & 0.023 & 0.005 \\
\hline \multicolumn{7}{|l|}{ Necrosis } \\
\hline Present vs Absent & $2.180(1.216-3.909)$ & 0.009 & 0.063 & $2.191(1.202-3.991)$ & 0.010 & 0.018 \\
\hline \multicolumn{7}{|l|}{ ECOG PS } \\
\hline $0 v s \geq 1$ & $2.123(1.333-3.382)$ & 0.002 & 0.004 & $2.356(1.426-3.893)$ & 0.001 & 0.002 \\
\hline \multicolumn{7}{|l|}{ CCL17 expression } \\
\hline High vs Low & $0.504(0.309-0.824)$ & 0.006 & 0.011 & $0.448(0.267-0.751)$ & 0.002 & 0.025 \\
\hline
\end{tabular}

ECOG PS Eastern Cooperative Oncology Group performance status, $H R$ hazard ratio, $\mathrm{Cl}$ confidence interval, OS overall survival, $R F S$ recurrence-free survival $P$-value $<0.05$ was regarded as statistically significant

tData obtained from the Cox proportional hazards model, ¥Bootstrapping with 1000 resamples were used

However, some limitations remained to be solved. This was a retrospective study in nature and the number of patients enrolled was limited. Though bootstrap internal validation has been used, the issues of over-fitting still existed. Cohort-specific biases including the method of tissue fixation, the staining protocols, the lot of the antibody, preparation and storage of the slides could largely affect our conclusions. Since all patients were from one institution, patient ethnicity/race, clinical practices at the institution and selection biases could also lead to cohort-specific biases thus affected the results. Lack of external validation was the major limitations of our research. We need to validate the results in an independent cohort to support our conclusions in the future. Moreover,

Table 3 Comparison of the predictive accuracy of the prognostic models

\begin{tabular}{|c|c|c|c|c|c|c|}
\hline \multirow[t]{2}{*}{ Models } & \multicolumn{3}{|l|}{ Overall survival } & \multicolumn{3}{|c|}{ Recurrence-free survival } \\
\hline & C-index $(95 \% \mathrm{Cl})$ & Coefficient $(95 \% \mathrm{Cl})$ & $P$-value & C-index $(95 \% \mathrm{Cl})$ & Coefficient $(95 \% \mathrm{Cl})$ & $P$-value \\
\hline CCL17 & $0.615(0.563-0.667)$ & & & $0.612(0.555-0.670)$ & & \\
\hline TNM & $0.706(0.652-0.760)$ & & & $0.658(0.601-0.719)$ & & \\
\hline TNM + CCL17 & $0.751(0.699-0.803)$ & $0.045(0.016-0.074)$ & $0.003+$ & $0.717(0.657-0.776)$ & $0.018(0.022-0.093)$ & $0.002+$ \\
\hline SSIGN & $0.632(0.580-0.685)$ & & & $0.674(0.617-0.731)$ & & \\
\hline SSIGN + CCL17 & $0.679(0.620-0.738)$ & $0.017(0.014-0.079)$ & $0.006+$ & $0.720(0.661-0.778)$ & $0.045(0.019-0.071)$ & $0.001+$ \\
\hline UISS & $0.735(0.688-0.781)$ & & & $0.710(0.658-0.762)$ & & \\
\hline UISS + CCL17 & $0.771(0.724-0.818)$ & $0.036(0.017-0.055)$ & $<0.001 \dagger$ & $0.752(0.697-0.802)$ & $0.047(0.018-0.065)$ & $0.001+$ \\
\hline Nomogram & $0.799(0.754-0.844)$ & & & $0.787(0.735-0.840)$ & & \\
\hline Nomogram vs SSIGN & & $0.167(0.118-0.215)$ & $<0.001 \neq$ & & $0.109(0.064-0.155)$ & $<0.001 \neq$ \\
\hline Nomogram vs UISS & & $0.064(0.030-0.099)$ & $<0.001 \neq$ & & $0.073(0.031-0.115)$ & $=0.001 \neq$ \\
\hline
\end{tabular}

C-index and $95 \% \mathrm{Cl}$ were calculated from 1000 bootstrap samples to protect from overfitting

C-index concordance index, CI confidence interval, SSIGN Mayo clinic stage, size, grade, and necrosis score, UISS UCLA Integrated Staging System

tCompared the c-index with the original model without CCL17 expression data; $¥$ Compared the c-index of nomogram with SSIGN/UISS stratification in different patient groups 


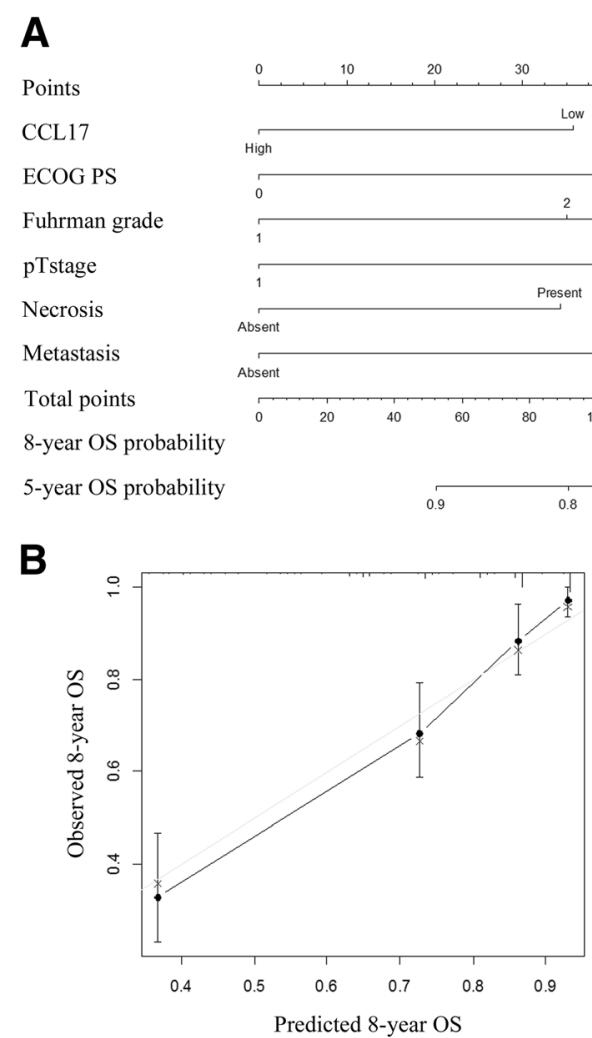

D

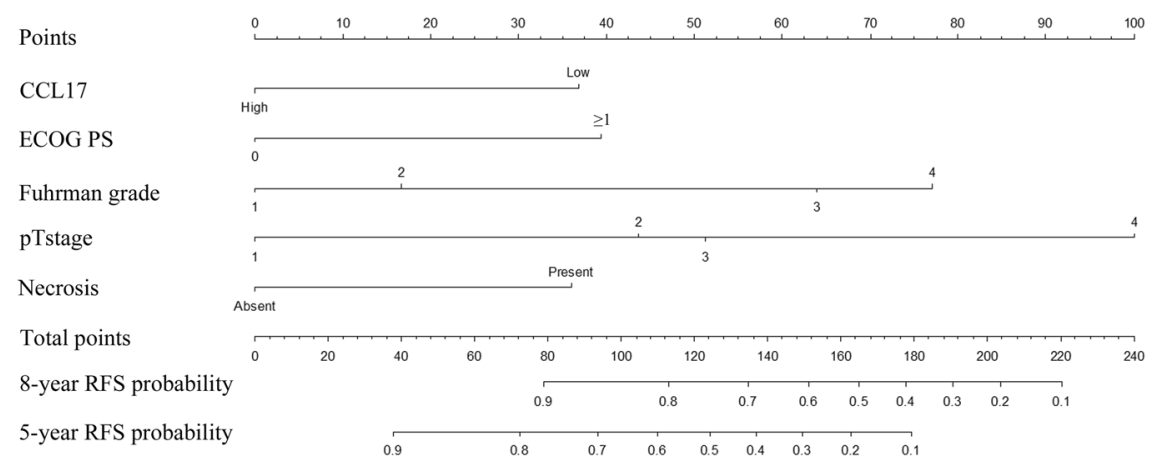

E

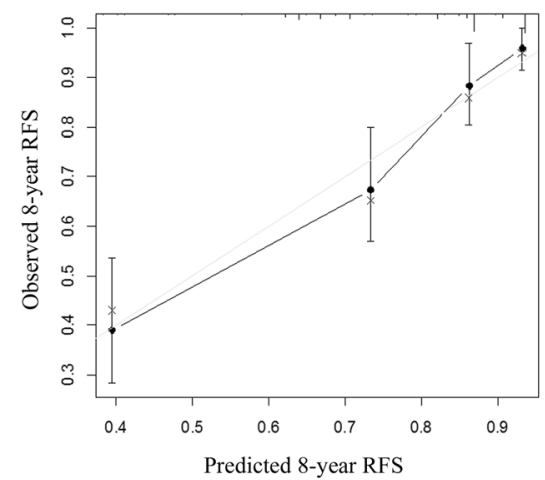

C

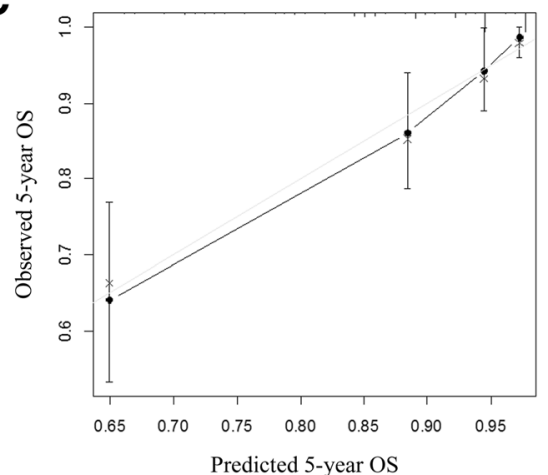

Points

F

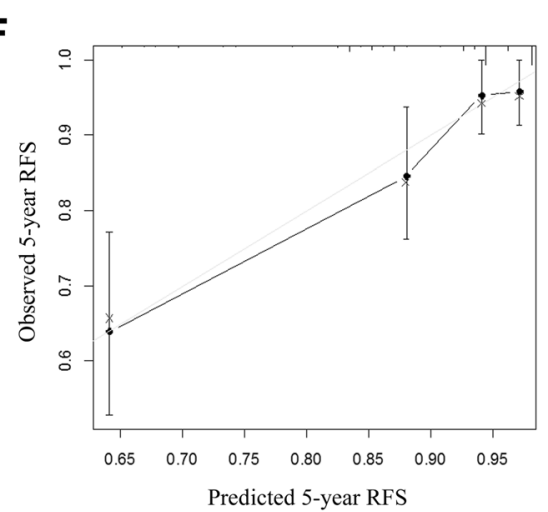

Fig. 3 (See legend on next page.) 
(See figure on previous page.)

Fig. 3 Prognostic nomograms and calibration plots for OS and RFS prediction. Six independent prognostic factors including CCL17 expression, ECOG PS, Fuhrman grade, pathological T stage, necrosis and metastasis were identified and entered into the nomogram (a). Calibration curves for predicting 8-year OS of CCRCC patients (b). Calibration curves for predicting 5-year OS of ccRCC patients(c). Five independent prognostic factors including CCL17 expression, ECOG PS, Fuhrman grade, pathological T stage and necrosis were identified and entered into the nomogram (d). Calibration curves for predicting 8-year RFS of cCRCC patients (e). Calibration curves for predicting 5-year RFS of ccRCC patients (f)

the heterogeneity of tumors might also affect the results though we took two tissue cores and took six shots from one tumor block. Further researches were required to investigate the roles of CCL17 in ccRCC tumor cells.

\section{Conclusion}

In conclusion, we have identified that low CCL17 expression was strongly associated with a poor outcome and CCL17 can be used as a novel prognostic factor in predicting patients' OS and RFS. We also developed nomograms for OS and RFS, which could give a better prediction for patients with ccRCC after surgery.

\section{Additional file}

Additional file 1: Table S1. Univariate analyses of characteristics associated with overall survival and recurrence-free survival. (DOC 58 kb)

\section{Abbreviations}

AJCC: American Joint Committee on Cancer; CCL17: chemokine ( $C-C$ motif) ligand 17; CCR4: CC chemokine receptor 4; CCR8: CC chemokine receptor 8; cCRCC: Clear cell renal cell carcinoma; ECOG PS: Eastern Cooperative Oncology Group performance status; OS: Overall survival; RFS: Recurrence-free survival; SSIGN: Mayo Clinic stage, size, grade and necrosis; TARC: Thymus and activationregulated chemokine; UISS: University of California Integrated Staging System

\section{Acknowledgments}

The authors would like to thank Dr. Yuan Ji, Dr. Jun Hou and Ms. Haiying Zeng (Department of Pathology, Zhongshan Hospital of Fudan University) for diagnosis confirmation and technical assistance, respectively.

\section{Funding}

This study was funded by grants from National Natural Science Foundation of China (31270863, 81471621, 81472227, 81472376 and 81671628), Program for New Century Excellent Talents in University (NCET-13-0146), Shanghai Municipal Natural Science Foundation (14ZR 1406300) and Zhongshan Hospital Science Foundation (2016ZSQN30). All these study sponsors have no roles in the study design, in the collection, analysis, and interpretation of data.

\section{Availability of data and materials}

The dataset supporting the conclusions of this article cannot be provided at present because we are collecting some other detailed follow up information. We will provide the original data by the time we finish the current task.

\section{Authors' contributions}

YX carried out and conducted experiments, performed statistical analysis, and drafted the manuscript. LL participated in the collection of patient materials and drafting of the manuscript. YX carried out laboratory work and data analysis. JW and WX performed laboratory work and participated in the correction of words in the manuscript. QB and YQ participated in the study design and collection of related articles. JX took charge of the study design and revising manuscript critically for important intellectual content. JG conceived of the study, and led the data analysis and oversaw the drafting of the manuscript. All authors read and approved the final manuscript.

\section{Competing interests}

The authors declare that they have no competing interests.

\section{Consent for publication}

Not applicable.

\section{Ethics approval and consent to participate}

The study was approved by Fudan University, Zhongshan Hospital research medical ethics committee. All specimens were obtained from patients with written informed consent.

Received: 11 June 2016 Accepted: 1 February 2017

Published online: 08 February 2017

\section{References}

1. Jemal A, Bray F, Center MM, Ferlay J, Ward E, Forman D. Global cancer statistics. CA Cancer J Clin. 2011;61(2):69-90

2. Ljungberg B, Bensalah K, Canfield S, Dabestani S, Hofmann F, Hora M, Kuczyk MA, Lam T, Marconi L, Merseburger AS, et al. EAU guidelines on renal cell carcinoma: 2014 update. Eur Urol. 2015;67(5):913-24.

3. Gupta K, Miller JD, Li JZ, Russell MW, Charbonneau C. Epidemiologic and socioeconomic burden of metastatic renal cell carcinoma (mRCC): a literature review. Cancer Treat Rev. 2008;34(3):193-205.

4. Meskawi M, Sun M, Trinh QD, Bianchi M, Hansen J, Tian Z, Rink M, Ismail S, Shariat SF, Montorsi $F$, et al. A review of integrated staging systems for renal cell carcinoma. Eur Urol. 2012;62(2):303-14.

5. Volpe A, Patard JJ. Prognostic factors in renal cell carcinoma. World J Urol. 2010;28(3):319-27

6. Vicari AP, Caux C. Chemokines in cancer. Cytokine Growth Factor Rev. 2002; 13(2):143-54.

7. Imai T, Baba M, Nishimura M, Kakizaki M, Takagi S, Yoshie O. The T celldirected CC chemokine TARC is a highly specific biological ligand for CC chemokine receptor 4. J Biol Chem. 1997;272(23):15036-42.

8. Kumai T, Nagato T, Kobayashi H, Komabayashi Y, Ueda S, Kishibe K, Ohkuri T, Takahara M, Celis E, Harabuchi Y. CCL17 and CCL22/CCR4 signaling is a strong candidate for novel targeted therapy against nasal natural killer/ T-cell lymphoma. Cancer Immunol Immunother. 2015;64(6):697-705.

9. Real E, Kaiser A, Raposo G, Amara A, Nardin A, Trautmann A, Donnadieu E. Immature dendritic cells (DCs) use chemokines and intercellular adhesion molecule (ICAM)-1, but not DC-specific ICAM-3-grabbing nonintegrin, to stimulate CD4+ T cells in the absence of exogenous antigen. J Immunol. 2004;173(1):50-60.

10. Semmling V, Lukacs-Kornek V, Thaiss CA, Quast T, Hochheiser K, Panzer U, Rossjohn J, Perlmutter P, Cao J, Godfrey DI, et al. Alternative cross-priming through CCL17-CCR4-mediated attraction of CTLS toward NKT cell-licensed DCs. Nat Immunol. 2010;11(4):313-20

11. Henry CJ, Ornelles DA, Mitchell LM, Brzoza-Lewis KL, Hiltbold EM. IL-12 produced by dendritic cells augments CD8+ T cell activation through the production of the chemokines CCL1 and CCL17. J Immunol. 2008:181(12): 8576-84.

12. Kim SP, Alt AL, Weight CJ, Costello BA, Cheville JC, Lohse C, Allmer C, Leibovich BC. Independent validation of the 2010 American Joint Committee on Cancer TNM classification for renal cell carcinoma: results from a large, single institution cohort. J Urol. 2011;185(6):2035-9.

13. Delahunt B, Cheville JC, Martignoni G, Humphrey PA, Magi-Galluzzi C, McKenney J, Egevad L, Algaba F, Moch H, Grignon DJ, et al. The International Society of Urological Pathology (ISUP) grading system for renal cell carcinoma and other prognostic parameters. Am J Surg Pathol. 2013; 37(10):1490-504.

14. Zisman A, Pantuck AJ, Wieder J, Chao DH, Dorey F, Said JW, de Kernion JB, Figlin RA, Belldegrun AS. Risk group assessment and clinical outcome 
algorithm to predict the natural history of patients with surgically resected renal cell carcinoma. J Clin Oncol. 2002;20(23):4559-66.

15. Leibovich BC, Blute ML, Cheville JC, Lohse CM, Frank I, Kwon ED, Weaver AL, Parker AS, Zincke H. Prediction of progression after radical nephrectomy for patients with clear cell renal cell carcinoma: a stratification tool for prospective clinical trials. Cancer. 2003:97(7):1663-71.

16. Frank I, Blute ML, Cheville JC, Lohse CM, Weaver AL, Zincke H. An outcome prediction model for patients with clear cell renal cell carcinoma treated with radical nephrectomy based on tumor stage, size, grade and necrosis: the SSIGN score. J Urol. 2002;168(6):2395-400.

17. Pan D, Xu L, Liu H, Zhang W, Zhu Y, Xu J, Gu J. Interleukin-11 receptor predicts post-operative clinical outcome in patients with early-stage clearcell renal cell carcinoma. Jpn J Clin Oncol. 2015;45(2):202-9.

18. Camp RL, Dolled-Filhart M, Rimm DL. X-tile: a new bio-informatics tool for biomarker assessment and outcome-based cut-point optimization. Clin Cancer Res. 2004;10(21):7252-9.

19. Meira-Machado L, Cadarso-Suarez C, Gude F, Araujo A. smoothHR: an R package for pointwise nonparametric estimation of hazard ratio curves of continuous predictors. Comput Math Methods Med. 2013;2013:745742.

20. Altman DG, Lausen B, Sauerbrei W, Schumacher M. Dangers of using "optimal" cutpoints in the evaluation of prognostic factors. J Natl Cancer Inst. 1994:86(11):829-35.

21. Bernardini G, Hedrick J, Sozzani S, Luini W, Spinetti G, Weiss M, Menon S, Zlotnik A, Mantovani A, Santoni A, et al. Identification of the CC chemokines TARC and macrophage inflammatory protein-1 beta as novel functional ligands for the CCR8 receptor. Eur J Immunol. 1998;28(2):582-8.

22. Sallusto F, Lenig D, Mackay CR, Lanzavecchia A. Flexible programs of chemokine receptor expression on human polarized T helper 1 and 2 lymphocytes. J Exp Med. 1998;187(6):875-83.

23. Ishida T, Ueda R. CCR4 as a novel molecular target for immunotherapy of cancer. Cancer Sci. 2006;97(11):1139-46.

24. Zhu F, Li X, Chen S, Zeng Q, Zhao Y, Luo F. Tumor-associated macrophage or chemokine ligand CCL17 positively regulates the tumorigenesis of hepatocellular carcinoma. Med Oncol. 2016;33(2):17.

25. Walter S, Weinschenk T, Stenzl A, Zdrojowy R, Pluzanska A, Szczylik C, Staehler M, Brugger W, Dietrich PY, Mendrzyk R, et al. Multipeptide immune response to cancer vaccine IMA901 after single-dose cyclophosphamide associates with longer patient survival. Nat Med. 2012;18(8):1254-61.

26. Weide B, Allgaier N, Hector A, Forschner A, Leiter U, Eigentler TK, Garbe C, Hartl D. Increased CCL17 serum levels are associated with improved survival in advanced melanoma. Cancer Immunol Immunother. 2015;64(9):1075-82.

27. Ness TL, Ewing JL, Hogaboam CM, Kunkel SL. CCR4 is a key modulator of innate immune responses. J Immunol. 2006;177(11):7531-9.

28. Ueda R. Clinical Application of Anti-CCR4 Monoclonal Antibody. Oncology. 2015;89 Suppl 1:16-21.

29. Weber C, Meiler S, Doring Y, Koch M, Drechsler M, Megens RT, Rowinska Z Bidzhekov K, Fecher C, Ribechini E, et al. CCL17-expressing dendritic cells drive atherosclerosis by restraining regulatory $\mathrm{T}$ cell homeostasis in mice. J Clin Invest. 2011;121(7):2898-910.

30. Heiseke AF, Faul AC, Lehr HA, Forster I, Schmid RM, Krug AB, Reindl W. CCL17 promotes intestinal inflammation in mice and counteracts regulatory T cell-mediated protection from colitis. Gastroenterology. 2012;142(2):335-45.

\section{Submit your next manuscript to BioMed Central and we will help you at every step:}

- We accept pre-submission inquiries

- Our selector tool helps you to find the most relevant journal

- We provide round the clock customer support

- Convenient online submission

- Thorough peer review

- Inclusion in PubMed and all major indexing services

- Maximum visibility for your research

Submit your manuscript at www.biomedcentral.com/submit

) Biomed Central 